\title{
Analogue Special and General Relativity by Optical Multilayer Thin Films: The Rindler Space Case
}

\author{
Sh. Dehdashti ${ }^{1}$, R. Roknizadeh ${ }^{1}$, A. Mahdifar ${ }^{2}$ \\ 1. Department of Physics, Quantum Optics Group, University of Isfahan, Isfahan, Iran \\ 2. Science Department, Shahrekord University,Shahrekord
}

June 20, 2018

\begin{abstract}
In this paper, to obtain an analogy between the curved spaces and the linear optics, we expand the idea of Ref. [1, 2] to the multilayer films. We investigate effects of thickness and index of refraction of the films on the Lorentzian transformations. In addition, by using the multilayer films, we suggest very simple experimental set-up which can serve as an analogue computer for testing special relativity. Finally, we draw an analogy between the Rindler space, as an example of the curved spaces, and a suitable multilayer film.
\end{abstract}

keywords: Multi-layers optics, Rindler space, Spinor map, Transfer Matrix.

\section{Introduction}

In recent years, analogies have taken on a major role in physics and mathematics [3, 4, 5]. They provide the new ways of looking at problems that permit cross-fertilization of ideas among different branches of science. Analogy methods have practical worth from two point of views: First, they provide new light on perplexing theoretical questions [3, 4]. Second, some of them are interesting for experimental set-up [3].

In this contribution we use the method developed in [1, 2], in a multilayer stack to show how we can set up an experimental model for special relativity and Rindler space as a special case of general relativity.

In the present section we review some of the relevant results achieved in [1, 2]. As it is well known, $S L(2, \mathbb{C}$ ) group is locally isomorphic (or a 2 to 1 homomorphic) to Lorentz

group, $S O(1,3)$. In fact, the transfer matrix, used in the classical optics for description of the interaction of light and matter, can be considered as an element of $S L(2, \mathbb{C})$ (see [6] and references therein). 
In ref. [2], authors gave a geometrical interpretation for the transfer matrix of an absorbing system. In fact, they considered an isolated absorbing film embedded between two identical ambient $I$ and substrate $I I$ media. A monochromatic, linear polarized plane wave falls from the ambient, as well as a plane wave of the same frequency and polarization falls from the substrate. If the amplitudes of the field are characterized by a column vector,

$$
\mathbf{E}=\left(\begin{array}{c}
E_{-} \\
E_{+}
\end{array}\right)
$$

where $E_{-}\left(E_{+}\right)$is denoted backward (forward)-travelling plane wave, and the amplitudes of the ambient and substrate are related by

$$
\mathbf{E}_{I}=\mathbf{M} \mathbf{E}_{I I}
$$

where $\mathbf{M}$ is the transfer matrix and is defined as,

$$
\mathbf{M}=\left(\begin{array}{cc}
\frac{T^{2}-R^{2}}{T} & \frac{R}{T} \\
-\frac{R}{T} & \frac{1}{T}
\end{array}\right),
$$

and $T$ and $R$ are the transmission and reflection coefficients, respectively.

By defining the coherency matrix [7] as

$$
\mathcal{E}=\left(\begin{array}{cc}
\left|E_{-}\right|^{2} & E_{-}^{*} E_{+} \\
E_{-} E_{+}^{*} & \left|E_{+}\right|^{2}
\end{array}\right)
$$

for both $I$ and $I I$, it is easy to show that,

$$
\mathcal{E}_{I}=M \mathcal{E}_{I I} M^{\dagger}
$$

It is useful to identify the variables $x^{\mu}$,

$$
x^{\mu}=\frac{1}{2} \operatorname{Tr}\left(\mathcal{E} \sigma^{\mu}\right)
$$

as the coordinates of a $(1+3)$-dimensional Minkowski space, in which $\sigma^{0}=\mathbb{I}$ (the identity) and $\sigma^{i}$ 's are the standard Pauli matrices. The conjugation (5) induces a transformation on the variables $x^{\mu}$ of the form

$$
x_{I}^{\mu}=T_{\nu}^{\mu}(M) x_{I I}^{\nu}
$$

where,

$$
T_{\nu}^{\mu}(M)=\frac{1}{2} \operatorname{Tr}\left(\sigma^{\mu} M \sigma_{\nu} M^{\dagger}\right)
$$

It is easy to see that the transformation $T(M)$ is a Lorentz transformation $[8]$ which is generated by $M$ and $-M$. Thus it is a $2 \rightarrow 1$ homomorphism map between $S L(2, \mathbb{C})$ and 
$S O(1,3)$.

We use the model which is developed in [1, 2] , and consider a monochromatic, linearly polarized plane electromagnetic wave incident on a non-absorption multilayer thin film. In this model basis coordinates are defined by the components of the electromagnetic wave, i.e., $E$ and $H$.

The organization of this paper is as follows. In section 2 we review the transfer matrix method for description of the multilayer films. In section 3 we introduce spinor map between $S L(2, \mathbb{C})$ and $S O(1,3)$, and we draw analogy between an EM field which passes through a multilayer film and transformation of coordinates under the Lorentzian group. In addition, two special cases, a multilayer, that is, quarter-wave films and a multilayer with very low thickness films, are studied in detail. In section 4 , by using the multilayer films, we suggest an experimental set-up for investigation of two kinematical properties, that is, time delay and length contraction as some of predictions of the special relativity. In section 5 we introduce an analogy between the Rindler space, as a curved space, and a suitable multilayer film. Finally, a summary and conclusion remarks are given in section 6.

\section{Matrix theory for description of the Multilayer films}

We consider an electromagnetic radiation falling onto a structure consisting of a thin film with uniform thickness $t$ and index of refraction $n$. This isolated (homogeneous and isotropic) film, is embedded between two media, $I$ and $I I$, with indexes of refraction, $n_{I}$ and $n_{I I}$, respectively. We consider a monochromatic, linearly polarized plane falling wave which is injected into the thin film from the above with amplitude $E_{I}$ and $H_{I}$. The field after passing the end layer is characterized by $E_{I I}$ and $H_{I I}$, as it is shown in Figure (11). We use transfer matrix method which is a method used in optics to analyse the propagation of electromagnetic through a stratified (layered) medium [7, 9].

The transfer-matrix method is based on the fact that, according to Maxwell's equations, there are simple continuity conditions for tangential component of electric field and normal component of magnetic field across boundaries.

In addition, this method can be developed to the multilayer films. 9]. For a stack with $m$ layers, the transfer-matrix can be defined as a product of matrices given by

$$
\mathcal{M}=M_{m} M_{m-1} \cdots M_{1}
$$

where the transfer-matrix of the $j^{\text {th }}$ layer, $M_{j}$, is given by

$$
M_{j}=\left(\begin{array}{cc}
\cos \delta_{j} & i \frac{\sin \delta_{j}}{\eta_{j}} \\
i \eta_{j} \sin \delta_{j} & \cos \delta_{j}
\end{array}\right),
$$


where $\delta_{j}=\frac{2 \pi}{\lambda} t_{j} n_{j} \cos \left(\theta_{j}\right)$ and $\eta_{j}$ for $s$-polarization and $p$-polarization is characterized by $\eta_{j}^{s}=n_{j} \cos \left(\theta_{j}\right)$ and $\eta_{j}^{p}=n_{j} / \cos \left(\theta_{j}\right)$ respectively [9]. It is obvious that the angle $\theta_{j}$ is related to the angle of incidence $\theta_{0}$ by Snell's law, that is,

$$
n_{j} \sin \theta_{j}=n_{0} \sin \theta_{0}
$$

\section{Lorentzian interpretation of the transfer matrix}

In this section, we use the spinor map, from $S L(2, \mathbb{C})$, onto the Lorentz group, $S O(1,3)$. We define a coherence matrix [7] as follows:

$$
\mathcal{E}=\left(\begin{array}{cc}
|E|^{2} & E^{*} H \\
E H^{*} & |H|^{2}
\end{array}\right)
$$

for both $I$ and $I I$, so that $\mathcal{E}_{I}$ and $\mathcal{E}_{I I}$ are related acording to the Eq. (5). We explicitly define the coordinates $x^{\mu}$, by using equation ([6)

$$
\begin{aligned}
& x^{0}=\frac{1}{2}\left(|E|^{2}+|H|^{2}\right), \quad x^{1}=\operatorname{Re}\left(E^{*} H\right), \\
& x^{2}=\operatorname{Im}\left(E^{*} H\right), \quad x^{3}=\frac{1}{2}\left(|E|^{2}-|H|^{2}\right) .
\end{aligned}
$$

It is worth to note that these coordinates behave as a light-like interval:

$$
\left(x^{0}\right)^{2}-\left(x^{1}\right)^{2}-\left(x^{2}\right)^{2}-\left(x^{3}\right)^{2}=0,
$$

Therefore, the conjugation relation (5) induces a transformation on the variables $x^{\mu}$ in the form of equation (7).

However, we can decompose the transfer matrix of a thin film, according to equations (9) and (10) with $j=1$, as

$$
M=\mathcal{B}^{-1} \mathcal{R} \mathcal{B}=\left(\begin{array}{cc}
\frac{1}{\sqrt{\eta}} & 0 \\
0 & \sqrt{\eta}
\end{array}\right)\left(\begin{array}{cc}
\cos \delta & i \sin \delta \\
i \sin \delta & \cos \delta
\end{array}\right)\left(\begin{array}{cc}
\sqrt{\eta} & 0 \\
0 & \frac{1}{\sqrt{\eta}}
\end{array}\right)
$$

Thus, under the homomorphism (8), $\mathcal{B}$ generates a boost of velocity $\beta=v$ (in natural units, where $c=1$ ), while $\mathcal{R}$ induces a pure spatial rotation.

To gain physical insights, we write explicitly the equation (8) for $\mathcal{B}$ and $\mathcal{R}$. Under the homomorphism (8), $\mathcal{R}$ generates the following matrix

$$
T(\mathcal{R}) \equiv R(2 \delta)=\left(\begin{array}{cccc}
1 & 0 & 0 & 0 \\
0 & 1 & 0 & 0 \\
0 & 0 & \cos 2 \delta & \sin 2 \delta \\
0 & 0 & -\sin 2 \delta & \cos 2 \delta
\end{array}\right)
$$


which is a rotation in the plane $x^{2}-x^{3}$ of angle twice the phase of the transmission coefficient.

Furthermore, diagonal matrix $\mathcal{B}$, under the homomorphism (8), generates a boost in the plane $x^{0}-x^{3}[10$,

$$
T(\mathcal{B}) \equiv L(0,0, \beta)=\left(\begin{array}{cccc}
\cosh \zeta & 0 & 0 & \sinh \zeta \\
0 & 1 & 0 & 0 \\
0 & 0 & 1 & 0 \\
\sinh \zeta & 0 & 0 & \cosh \zeta
\end{array}\right)
$$

where rapidity $\zeta$ is obtained from the relations

$$
\begin{aligned}
& \cosh \zeta=\frac{1}{2}\left(\eta+\frac{1}{\eta}\right), \\
& \sinh \zeta=\frac{1}{2}\left(\eta-\frac{1}{\eta}\right),
\end{aligned}
$$

and velocity $\beta$ is defined by

$$
\beta=\tanh \zeta=\frac{\left(\eta-\frac{1}{\eta}\right)}{\left(\eta+\frac{1}{\eta}\right)}
$$

In Figure (2), we have plotted the variation of $\beta$ with respect to the $\theta$ for different values of the index of refraction, $n$, when the electromagnetic field have $s$-, and $p$-polarization. It is seen that for every values of the index of refraction, $\beta$ has maximum or minimum in $\theta=0$. In addition, for a fixed $\theta$, the $\beta$ is increased by increasing the refraction index, for both $s$-, and $p$-polarization. However, for the large values of the refraction index, $n \gg 1$, for both polarization, the result is the same, as shown in Figure (2). Finally, by using equations (15), (16) and (17) we obtain $T(M)$ as

$$
T(M)=L(0,0,-\beta) R(2 \omega t) L(0,0, \beta)
$$

where $\omega=\delta / t$. Also, with some calculations, we can rewrite equation (21), in the following form [11, 12].

$$
T(M)=R(2 \omega t) L(0, \beta \sin (2 \omega t),-\beta \cos (2 \omega t)) L(0,0, \beta) .
$$

In addition, by using Einstein rule for the composition of velocities [13],

$$
\vec{\beta}_{1} \oplus \vec{\beta}_{2}=\frac{1}{1+\vec{\beta}_{1} \vec{\beta}_{2}}\left\{\vec{\beta}_{1}+\vec{\beta}_{2}+\frac{\gamma_{\beta_{1}}}{1+\gamma_{\beta_{1}}}\left(\vec{\beta}_{1} \times\left(\vec{\beta}_{1} \times \vec{\beta}_{2}\right)\right)\right\},
$$

we can write $T(M)$ as a rotation and a boost,

$$
T(M)=R(2 \omega t) L\left(0, \beta_{2}^{\prime}, \beta_{3}^{\prime}\right)
$$


where

$$
\begin{aligned}
& \beta_{2}^{\prime}=\frac{\beta \sin (2 \omega t)}{1-\beta^{2} \cos (2 \omega t)}\left[1-\frac{\beta^{2} \cos (2 \omega t)}{1+\sqrt{1-\beta^{2}}}\right] \\
& \beta_{3}^{\prime}=-\frac{\beta}{1-\beta^{2} \cos (2 \omega t)}\left(\cos (2 \omega t)+\frac{\beta^{2} \sin ^{2}(2 \omega t)}{1+\sqrt{1-\beta^{2}}}\right)
\end{aligned}
$$

In Figures (3) and (4), we have plotted the variations of $\beta_{2}^{\prime}, \beta_{3}^{\prime}$ and $\left|\beta^{\prime}\right|=\sqrt{\beta_{2}^{\prime 2}+\beta_{3}^{\prime 2}}$ with respect to $t$ for $n=2.00049$ and $\theta=\pi / 9$, for $s$-polarization and $p$-polarization, respectively. As it is seen, $\beta_{2}^{\prime}, \beta_{3}^{\prime}$ and $\left|\beta^{\prime}\right|$ depend periodically on $t$.

We now turn to the multi-layer stack with $m$ films. By employing the method which is used to obtain equation (22), and by using equation (24), we can write $T(\mathcal{M})$ as

$$
T(\mathcal{M})=R(2 \Omega t) L\left(0, \mathcal{B}_{2}^{(m)}, \mathcal{B}_{3}^{(m)}\right) \cdots L\left(0, \mathcal{B}_{2}^{(1)}, \mathcal{B}_{3}^{(1)}\right),
$$

where $\Omega=\sum_{i=1}^{m} \omega^{(i)}$ and

$$
\begin{aligned}
& \mathcal{B}_{2}^{(i)}=\beta_{2}^{(i)} \cos \left(2 \Omega^{(i)} t^{(i)}\right)-\beta_{3}^{(i)} \sin \left(2 \Omega^{(i)} t^{(i)}\right), \\
& \mathcal{B}_{3}^{(i)}=\beta_{2}^{(i)} \sin \left(2 \Omega^{(i)} t^{(i)}\right)+\beta_{3}^{(i)} \cos \left(2 \Omega^{(i)} t^{(i)}\right),
\end{aligned}
$$

with $\Omega^{(i)}=\sum_{j=1}^{i-1} \omega^{(j)}$ and

$$
\begin{aligned}
& \beta_{2}^{(i)}=\frac{\beta^{i} \sin \left(2 \omega^{i} t^{i}\right)}{1-\left(\beta^{i}\right)^{2} \cos \left(2 \omega^{i} t^{i}\right)}\left[1-\frac{\left(\beta^{i}\right)^{2} \cos \left(2 \omega^{i} t^{i}\right)}{1+\sqrt{1-\left(\beta^{i}\right)^{2}}}\right] \\
& \beta_{3}^{(i)}=-\frac{\beta^{(i)}}{1-\left(\beta^{(i)}\right)^{2} \cos \left(2 \omega^{(i)} t^{(i)}\right)}\left(\cos \left(2 \omega^{(i)} t^{(i)}\right)+\frac{\left(\beta^{(i)}\right)^{2} \sin ^{2}\left(2 \omega^{(i)} t^{(i)}\right)}{1+\sqrt{1-\left(\beta^{(i)}\right)^{2}}}\right) .
\end{aligned}
$$

Also, by using Einstein addition rule (23), we can rewrite equation (26) as

$$
T(\mathcal{M})=R(2 \Omega t) L\left(0, \mathcal{B}_{2}^{(\mathcal{M})}, \mathcal{B}_{3}^{(\mathcal{M})}\right)
$$

where $\mathcal{B}_{2}^{(\mathcal{M})}$ and $\mathcal{B}_{3}^{(\mathcal{M})}$ are given by applying the rule $(\underline{23})$ consecutively.

\subsection{A multilayer with quarter-wave films}

In this section, we consider a multilayer with quarter-wave films [14], that is $t_{j} n_{j}=\lambda / 4$ and assume that $\theta_{0}=0$, so that $\cos \delta_{j}=0$ and $\sin \delta_{j}=1$. As an example, for a stack consists of two layers, matrix $M$, is given by,

$$
M=\left(\begin{array}{cc}
-\frac{n_{1}}{n_{2}} & 0 \\
0 & -\frac{n_{2}}{n_{1}}
\end{array}\right),
$$


which under the homomorphism (8), generates a boost as [10],

$$
T(M) \equiv L\left(0,0, \beta_{q w}^{(2)}\right)=\left(\begin{array}{cccc}
\cosh \zeta_{q w}^{(2)} & 0 & 0 & \sinh \zeta_{q w}^{(2)} \\
0 & 1 & 0 & 0 \\
0 & 0 & 1 & 0 \\
\sinh \zeta_{q w}^{(2)} & 0 & 0 & \cosh \zeta_{q w}^{(2)}
\end{array}\right)
$$

where velocity $\beta_{q w}^{(2)}$ is given by

$$
\beta_{q w}^{(2)}=\tanh \zeta_{q w}^{(2)}=\frac{1-\left(\frac{n_{2}}{n_{1}}\right)^{4}}{1+\left(\frac{n_{2}}{n_{1}}\right)^{4}},
$$

and rapidity $\zeta_{q w}^{(2)}$ is obtained from the relations

$$
\begin{aligned}
\cosh \zeta_{q w}^{(2)} & =\frac{1}{2}\left(\left(\frac{n_{1}}{n_{2}}\right)^{2}+\left(\frac{n_{2}}{n_{1}}\right)^{2}\right), \\
\sinh \zeta_{q w}^{(2)} & =\frac{1}{2}\left(\left(\frac{n_{1}}{n_{2}}\right)^{2}-\left(\frac{n_{2}}{n_{1}}\right)^{2}\right) .
\end{aligned}
$$

In general, for a stack consists of $2 m$ quarter-wave layers, in which the thickness of $j^{\text {th }}$ layer is $t_{j}=\frac{\lambda}{4 n_{j}}, j=1,2, \cdots, 2 m$, after some simple calculation, we can achieve $T\left(M_{2 m}\right)$ as,

$$
T\left(M_{2 m}\right) \equiv L\left(0,0, \beta_{q w}^{(2 m)}\right)=\left(\begin{array}{cccc}
\cosh \zeta_{q w}^{(2 m)} & 0 & 0 & \sinh \zeta_{q w}^{(2 m)} \\
0 & 1 & 0 & 0 \\
0 & 0 & 1 & 0 \\
\sinh \zeta_{q w}^{(2 m)} & 0 & 0 & \cosh \zeta_{q w}^{(2 m)}
\end{array}\right)
$$

where velocity $\beta_{q w}^{(2 m)}$ is calculated by the rule (23). As an example for $2 m=4$, we have

$$
\beta_{q w}^{(4)}=\tanh \zeta_{q w}^{(4)}=\frac{\beta_{1}+\beta_{2}}{1+\beta_{1} \beta_{2}} .
$$

\section{$3.2 \quad$ A multilayer with very low thickness}

In this section we study a multilayer, with $m$ layers, in such a way that $\eta^{(i)}$ 's are near to the unit, $\eta^{(i)} \simeq 1$. Thus, by using equation (20) and keeping only first-order term of $\eta^{(i)}$, we obtain $\beta^{(i)}=-1+\eta^{(i)}$ and therefore the Lorentzian transformations are replaced by the Galilean transformations. Thus, by these assumption, we can rewrite the equation (31) as the following relation:

$$
T(\mathcal{M})=R(2 \Omega t) L\left(0, \mathcal{B}_{2}^{(\mathcal{M})}, \mathcal{B}_{3}^{(\mathcal{M})}\right)
$$


where

$$
\begin{aligned}
& \mathcal{B}_{2}^{(\mathcal{M})}=\sum_{i=1}^{m} \beta^{(i)} \sin \left[2\left(\omega^{(i)}+\Omega^{(i)}\right) t^{(i)}\right] \\
& \mathcal{B}_{3}^{(\mathcal{M})}=\sum_{i=1}^{m} \beta^{(i)} \cos \left[2\left(\omega^{(i)}+\Omega^{(i)}\right) t^{(i)}\right] .
\end{aligned}
$$

In addition, when we assume $t$ is very thin, from equation (21), by keeping only firstorder terms in $t$, the rotation $R(2 \omega t)$ appears as a pure boost, $L(0,0, \beta)$, which is followed by another pure boost in another direction, $L(0,2 a d t, 0)$, so that the result is like a Thomas precession. That is, we can write [10, 15],

$$
R(2 \omega d t)=L(0,-2 a d t,-\beta) L(0,2 a d t, 0) L(0,0, \beta),
$$

where $\omega=\frac{2 \pi}{\lambda} n \cos \theta_{I I}$ and $a=-\frac{\omega}{\gamma}$ is the acceleration along the second axis, $x^{2}$.

\section{Experimental Set-up of Time Delay and Length Contrac- tion by Thin Films}

We can now propose some specific experiments bases on the properties discussed in the preceding sections. The basic experimental set-up consists essentially of a monochromatic plane wave, linear polarized ( $s$ or $p$ ) with the specific values of $|E|$ and $|H|$.

We use a stack consists of 2 quarter-wave layers. According to the equation (33), the coordinates $x^{1}$ and $x^{2}$ under the action of transformation matrix remain invariant. Therefore, it is possible to consider $T(\mathcal{M})$ as a boost which belongs to $S O(1,1)$ :

$$
\left(\begin{array}{c}
x_{\text {out }}^{0} \\
x_{\text {out }}^{3}
\end{array}\right)=\frac{1}{2}\left(\frac{n_{1}}{n_{2}}\right)^{2}\left(\begin{array}{cc}
1+\left(\frac{n_{2}}{n_{1}}\right)^{4} & 1-\left(\frac{n_{2}}{n_{1}}\right)^{4} \\
1-\left(\frac{n_{2}}{n_{1}}\right)^{4} & 1+\left(\frac{n_{2}}{n_{1}}\right)^{4}
\end{array}\right)\left(\begin{array}{c}
x_{\text {in }}^{0} \\
x_{\text {in }}^{3}
\end{array}\right) .
$$

To show how the experimental set-up works, let us suppose that the electromagnetic fields, $E_{\text {in }}$ and $H_{\text {in }}$, fall onto the multi-layer. From equation (13) we have

$$
x_{i n}^{0}=\frac{1}{2}\left(\left|E_{i n}\right|^{2}+\left|H_{i n}\right|^{2}\right), \quad x_{i n}^{3}=\frac{1}{2}\left(\left|E_{i n}\right|^{2}-\left|H_{i n}\right|^{2}\right),
$$

and

$$
\begin{aligned}
& x_{\text {out }}^{0}=\frac{1}{2}\left(\left|E_{\text {out }}\right|^{2}+\left|H_{\text {out }}\right|^{2}\right)=\frac{1}{2}\left(\frac{n_{1}}{n_{2}}\right)^{2}\left\{\left|E_{\text {in }}\right|^{2}+\left(\frac{n_{2}}{n_{1}}\right)^{4}\left|H_{\text {in }}\right|^{2}\right\}, \\
& x_{\text {out }}^{3}=\frac{1}{2}\left(\left|E_{\text {out }}\right|^{2}-\left|H_{\text {out }}\right|^{2}\right)=\frac{1}{2}\left(\frac{n_{1}}{n_{2}}\right)^{2}\left\{\left|E_{\text {in }}\right|^{2}-\left(\frac{n_{2}}{n_{1}}\right)^{4}\left|H_{\text {in }}\right|^{2}\right\} .
\end{aligned}
$$

These equations clearly show that there are many possible experimental methods to choose the input fields in such a way that two different points in a reference frame may be appear 
in the other frame as either with the same temporal or same spatial coordinate.

We consider two experimental set-ups with different inputs $E_{i n(j)}$ and $H_{i n(j)}$ and corresponding to outputs $E_{\text {out }(j)}$ and $H_{\text {out }(j)}$,where $j=1,2$. Thus, it is easy to show that $x_{\text {out }(1)}^{0}=x_{\text {out }(2)}^{0}$ when

$$
\left|E_{i n(2)}\right|^{2}-\left|E_{i n(1)}\right|^{2}=\left(\frac{n_{2}}{n_{1}}\right)^{4}\left\{\left|H_{i n(1)}\right|^{2}-\left|H_{i n(2)}\right|^{2}\right\}
$$

and $x_{\text {out }(1)}^{3}=x_{\text {out }(2)}^{3}$ when

$$
\left|E_{i n(2)}\right|^{2}-\left|E_{i n(1)}\right|^{2}=\left(\frac{n_{2}}{n_{1}}\right)^{4}\left\{\left|H_{i n(2)}\right|^{2}-\left|H_{i n(1)}\right|^{2}\right\} .
$$

By using equation (45), the first set-up can be used to demonstrate the length contraction,

$$
l_{\text {out }} \equiv x_{\text {out }(2)}^{3}-x_{\text {out }(1)}^{3}=\frac{1}{\gamma}\left(x_{\text {in }(2)}^{3}-x_{\text {in }(1)}^{3}\right) .
$$

In order to put forward the phenomenon of the time dilation, let us consider another experimental set-up in which $E_{i n(1)}, H_{i n(1)}, E_{i n(2)}$ and $H_{i n(2)}$ satisfy the equation (46). Therefore, measurement of $\Delta t_{\text {out }} \equiv x_{\text {out (2) }}^{0}-x_{\text {out (1) }}^{0}$ may be regarded as a time interval measurement. Therefore, we obtain

$$
\Delta t_{\text {out }} \equiv x_{\text {out }(2)}^{0}-x_{\text {out }(1)}^{0}=\frac{1}{\gamma}\left(x_{\text {in }(2)}^{0}-x_{\text {in }(1)}^{0}\right) .
$$

In summary, we have proposed a series of specific experiments by using a multilayer, which contains two films and the thickness of their films are given by $t_{j} n_{j}=\lambda / 4$, that clearly demonstrate the most important predictions of the Lorentz kinematics.

\section{A Multi-layer as a Rindler Space}

Maxwell's equations in general coordinates are written as [5]

$$
\left(\sqrt{g} g^{i j} E_{j}\right)_{, i}=\frac{\sqrt{g} \rho}{\varepsilon_{0}}, \quad\left(\sqrt{g} g^{i j} B_{j}\right)_{, i}=0,
$$

and

$$
[i j k] E_{k, j}=\frac{\partial\left(\sqrt{g} g^{i j} B_{j}\right)}{\partial t}, \quad[i j k] H_{k, j}=\frac{1}{c^{2}} \frac{\partial\left(\sqrt{g} g^{i j} E_{j}\right)}{\partial t}+\mu_{0} \sqrt{g} j^{i}
$$

where permutation symbol, $[i j k]$, is defined by

$$
[i j k]=\left\{\begin{array}{cc}
+1, & \text { if } i j k \text { is an even permutation of } 123 \\
-1, & \text { if } i j k \text { is an odd permutation of } 123 \\
0, & \text { otherwise }
\end{array}\right.
$$


and the Einstein summation convention is used. As it is well known in Optics, the Maxwell's equations in empty-curved space can be replaced by Maxwell's equations in the flat space which is filled by dielectric media with the following parameters [5],

$$
\varepsilon^{i j}=\mu^{i j}= \pm \sqrt{g} g^{i j} .
$$

Therefore, we can see a correspondence between geometry and matter, as in the general relativity. Thus, in this case, the index of refraction is obtained by [5]

$$
n^{2}=\left(\begin{array}{lll}
\varepsilon_{11} \varepsilon_{22}-\varepsilon_{12} \varepsilon_{21} & \varepsilon_{13} \varepsilon_{32}-\varepsilon_{12} \varepsilon_{33} & \varepsilon_{12} \varepsilon_{23}-\varepsilon_{13} \varepsilon_{22} \\
\varepsilon_{23} \varepsilon_{31}-\varepsilon_{21} \varepsilon_{33} & \varepsilon_{11} \varepsilon_{33}-\varepsilon_{13} \varepsilon_{31} & \varepsilon_{13} \varepsilon_{21}-\varepsilon_{13} \varepsilon_{23} \\
\varepsilon_{21} \varepsilon_{12}-\varepsilon_{22} \varepsilon_{31} & \varepsilon_{12} \varepsilon_{31}-\varepsilon_{11} \varepsilon_{32} & \varepsilon_{11} \varepsilon_{22}-\varepsilon_{12} \varepsilon_{21}
\end{array}\right)
$$

Now, if we consider the metric,

$$
d s^{2}=-d t^{2}+N^{2}(x)\left(d x^{2}+d y^{2}+d z^{2}\right),
$$

by using equations (52) and (53), the index of refraction is given by $n(x)=[N(x)]^{-1}$.

On the other hand, the principle of equivalence make the gravitational field connect to the acceleration. Therefore, to study gravitational effects, we can generalize concept of Lorentz transformations so as to include observers who are moving with a constant acceleration with respect to an inertial frame. This metric is called Rindler metric and described as [16,

$$
d s^{2}=-(g x)^{2} d t^{2}+d x^{2}+d y^{2}+d z^{2} .
$$

It is easy to see that the metrics (54) and (55) are conformal. Accordingly, by using the equation (53), we obtain the refractive index of the analogue media with the Rindler space as the following relation:

$$
n=g x .
$$

Thus, for analogue Rindler space we assume a multilayer, for which the refractive index of the $j^{\text {th }}$ layer is characterized by,

$$
n_{j}=g\left(j-\frac{1}{2}\right) t
$$

However, up to the first order approximation in $\beta^{(i)}$ and by using equation (57), for $s$ - and $p$-polarization, when $\theta_{0}=0$, we can rewrite equation (39) as,

$$
T(\mathcal{M})=L\left(0, \mathbb{B}_{2}, \mathbb{B}_{3}\right)
$$

where

$$
\mathbb{B}_{2}=\sum_{i=0}^{m} \frac{\left(g\left(j-\frac{1}{2}\right) t\right)^{2}-1}{\left(g\left(j-\frac{1}{2}\right) t\right)^{2}+1} \sin \left(\frac{2 \pi}{\lambda} t^{2} i^{2}\right)
$$


and

$$
\mathbb{B}_{3}=\sum_{i=0}^{m} \frac{\left(g\left(j-\frac{1}{2}\right) t\right)^{2}-1}{\left(g\left(j-\frac{1}{2}\right) t\right)^{2}+1} \cos \left(\frac{2 \pi}{\lambda} t^{2} i^{2}\right) .
$$

Also, we can consider a stack consists of the quarter-wavelength layers, i.e., $t_{j} n_{j}=\lambda / 4$. Therefore, by using the equation (56), the thickness of $j^{\text {th }}$ layer is

$$
t_{j}=\sqrt{\frac{\lambda}{2 g}+x_{j}^{2}}-x_{j}, \quad x_{j}=\sum_{i=1}^{j-1} t_{i} .
$$

After some calculation, we can obtain thickness of the $j^{\text {th }}$ layer as,

$$
t_{j}=\sqrt{\frac{\lambda}{2 g}}(\sqrt{j}-\sqrt{j-1}),
$$

and the index of refraction of this layer is obtained by

$$
n_{j}=\sqrt{\frac{\lambda g}{8}}(\sqrt{j}+\sqrt{j-1}) .
$$

We can now construct an analogy between the Rindler space and a multilayer with $2 m$ quarter-wavelength layers, in which the thickness of $j^{\text {th }}$ layer is given by equation (62) and its refraction index is given by equation (63). Accordingly we obtain ,

$$
E_{\text {Multilyer }}=\frac{\sqrt{j+1}+\sqrt{j}}{\sqrt{j}+\sqrt{j-1}} E_{0}
$$

In Refs. [17, 18, electrodynamics in the Rindler space had been studied by using a phenomenological approach. Accordingly, the component of the electric field, near the horizon, $x=0$, reads as follow:

$$
E_{\text {Rindler }}(x)=E_{0} K_{i 2 \pi / \lambda}\left(\frac{2 \pi}{\lambda} x\right),
$$

where $K_{i 2 \pi / \lambda}(2 \pi x / \lambda)$ is the modified Bessel function of order $i 2 \pi / \lambda$.

Figure (5) displays a comparison between the output values of the electric fields are given by equation (64) and equation (65), i.e.,

$$
\Delta E=\operatorname{Abs}\left(|E|_{\text {Multilayer }}-|E|_{\text {Rindler }}\right),
$$

in terms of $x=\sqrt{\frac{\lambda}{2 g} j}$ and for different values of $\lambda$. As it is seen, by increasing $x$ (increasing the number of layers), $\Delta E$ approaches to zero. In addition, $\Delta E$ is decreased more rapidly by decreasing the $\lambda$. 


\section{6 conclusion}

This paper is an example of analogue models of Einstein Relativity. Firstly, we have used a geometrical tool to analyze a multilayer film in a concise way that is closely related to the special relativity. Then, this method was made an analogy between multilayer stack and curved space in the general relativity. In fact, in the first step, we have seen how the transfer matrix can be interpreted as multiplication of a boost, a rotation and another boost.

In addition, in this case, we have suggested an experimental set-up which tests the most important predictions of the Lorentz kinematics, i.e., time delay and length contraction. However, by using this isomorphism, apart from a relativistic presentation of the topic, which has interest in its own, we suggested an experimental set-up which is, in theoretical view, so simple and understandable, and, in practical level, was so easy to accomplish, in contrast to the similar experimental set-up [19].

Finally, we have employed analogue method in replacing Maxwell's equations in emptycurved space to Maxwell's equations in the flat space where the later is filled by a media. Then, we have applied this method to the Rindler frame, which by using principle of equivalence, is locally equivalent to gravitational fields. This means that this method makes an analogy between accelerated frame in general relativity and a suitable multilayer films, with specific values of the refractive indexes and thickness of the films.

\section{Acknowledgement}

The authors wish to thank The Office of Graduate Studies of The University of Isfahan and Shahrekord University for their support.

\section{References}

[1] Sánchez-Soto, L. L.; Monzón, J. J.; Barriuso, A. G.; Cariñena, J. F. Phys. Rep. 2012, 513, 191.

[2] Monzón, J. J.; Barriuso, A. G.; Sánchez-Soto, L. L.; Montesinos-Amilibia, J. M. Phys. Rev. A 2011, 84, 023830.

[3] Novello, M.; Visser, M.; Volovik, G. Artificial Blackholes; World Scientific, Singapore, 2002.

[4] Leonhardt, U. Nature (London) 2002, 415, 406.

[5] Leonhardt, U.; Philbin, T. G. Geometry and Light: The Science of Invisibility; Dover, Mineola, 2010. 
[6] Yonte, T.; Monzón, J. J.; Sánchez-Soto, L. L.; Cariñena, J. F.; López-Lacasta, C. J. Opt. Soc. Am. A 2002, 19, 603.

[7] Born, M.; Wolf, E. Principles of optics: electromagnetic theory of propagation, interference and diffraction of light; Oxford, Pergamon Press, 1964.

[8] Naber, G. L. The Geometry of Minkowski Spacetime; Springer, New York, 1992.

[9] Bass, M.; Li, G. Handbook of Optics, Volume IV; McGraw-Hill, New York, 2010.

[10] Misner, Ch. W.; Thorne, K. S.; Wheeler, J. A. Gravitation; W. H. Freeman, New York, 1973.

[11] Sattinger, D. H.; Weaver, O. L. Lie Groups and Algebras with Applications to Physics, Geometry, and Mechanics; Springer-Verlag, 1993.

[12] Cornwell, J. F. Group theory in physics, Volume I; Academic Press, 1989.

[13] Ungar, A. A. Comput. Math. Appl. 2007, 53, 1228.

[14] Fowles, G. R. Introduction to Modern Optics; Dover Publications, USA, 1986.

[15] O’Donnell, K.; Visser, M. Eur. J. Phys. 2011, 32, 1033.

[16] Padmanabhan, T. Gravitation: Foundations and Frontiers; Cambridge University Press, Cambridge, 2010.

[17] Brevik, I. J. Math. Phys. 1987, 28, 2241.

[18] Hacyan, Sh. J. Opt. 2011, 13, 105710.

[19] Monzón, J. J.; Sánchez-Soto, L. L. Phys. Lett A 1999, 262, 18. 


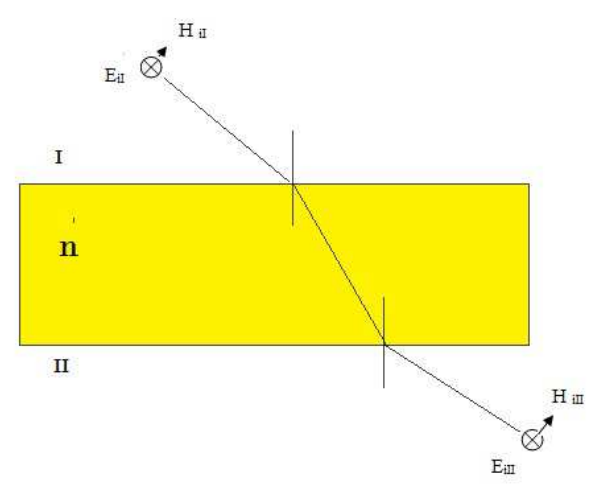

Figure 1: Scheme of the input and output field in a thin film.

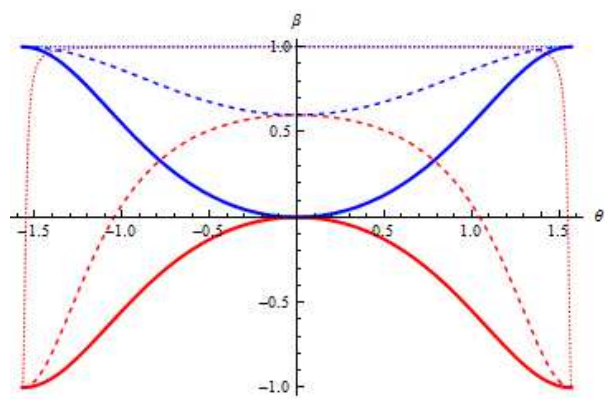

Figure 2: (Colour online) The variation of $\beta$, for $s$ and $p$-polarization, with respect to $\theta$ for different values of the index of refraction, $n=1$ are plotted by red and blue line, $n=2$ by red and blue dashing line and $n=10$ by red and blue dot line, for $s$ and $p$-polarization, respectively.

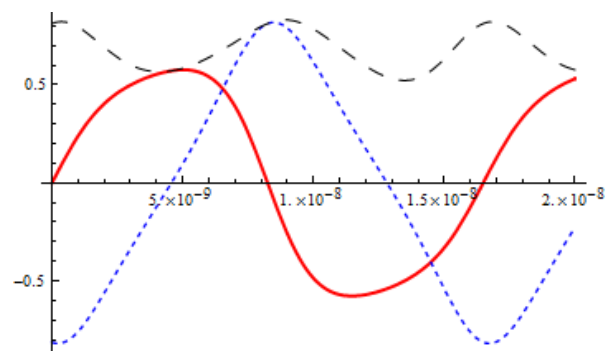

Figure 3: (Colour online) The variation of $\beta_{2}^{\prime}$ by red line, $\beta_{3}^{\prime}$ by blue dot line, and $\left|\beta^{\prime}\right|$ by black dashing line with respect to $t$ for the fixed index of refraction, $n=2.419$, for $s$-polarization. 


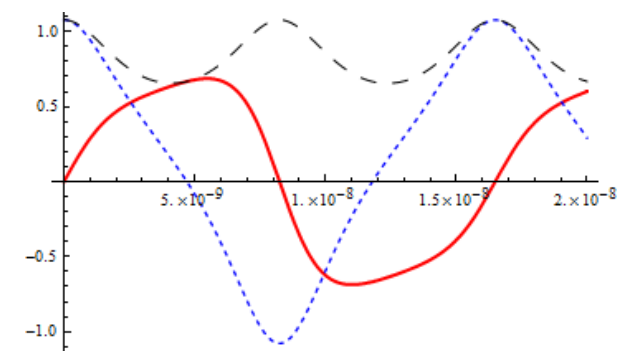

Figure 4: (Colour online) The variation of $\beta_{2}^{\prime}$ by red line, $\beta_{3}^{\prime}$ by blue dot line, and $\left|\beta^{\prime}\right|$ by black dashing line with respect to $t$ for the fixed index of refraction, $n=2.419$, for p-polarization

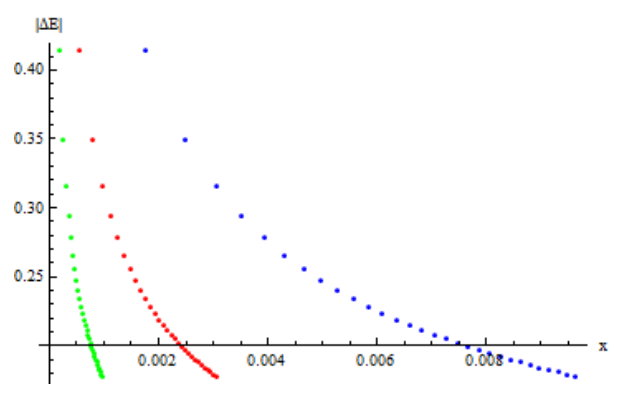

Figure 5: (Colour online) The variation of $\Delta E$ with respect to $x=\sqrt{\frac{\lambda}{2 g} j}$ with $g=1$ and $2 m=60$ for $\lambda=0.06199 \times 10^{-6}, \lambda=0.06199 \times 10^{-5}$ and $\lambda=0.06199 \times 10^{-4}$ by green ( left), red ( middle) and blue (right) points, respectively. 